\title{
Cardiodoron $^{\circledR}$ bei Patienten mit Schlafstörungen - Ergebnisse einer prospektiven Beobachtungsstudie
}

\author{
Claudia Rother Martin Schnelle
}

WELEDA AG, Klinische Forschung, Schwäbisch Gmünd, Deutschland

\author{
Schlüsselwörter \\ Schlafstörungen · Prospektive, multizentrische \\ Beobachtungsstudie · Primula - Hyoscyamus - Onopordum
}

\section{Zusammenfassung}

Hintergrund: Schlafstörungen gehören zu den häufigsten gesundheitlichen Problemen der heutigen Zeit. Stress und die dadurch bedingte innere Anspannung sowie eine unrhythmische Lebensführung z.B. durch Schichtarbeit sind bekannte auslösende Faktoren. Weniger bekannt ist, dass auch funktionelle Herz-Kreislauf-Beschwerden zu Störungen des Schlafs führen können und dass deren Behandlung zu einer Verbesserung der Schlafqualität beiträgt. Ganzheitlich betrachtet geht es daher um die Wiederherstellung einer gesunden Rhythmik, insbesondere des Herz-/Atem- sowie des Schlaf-Wach-Rhythmus, die Cardiodoron ${ }^{\circledR}$, eine Heilpflanzenkomposition aus Primula veris, Hyoscyamus niger und Onopordum acanthium, unterstützt. Patienten und Methoden: Mittels einer prospektiven, multizentrischen Beobachtungsstudie sollte ermittelt werden, wie sich funktionelle Herz-Kreislauf-Beschwerden und/oder Schlafstörungen unter der Behandlung mit Cardiodoron ${ }^{\circledR}$ (Dilution) über 3-6 Monate entwickeln. Im Zeitraum von September 2009 bis März 2012 dokumentierten 92 Ärzte 501 Patienten, von denen 380 über Schlafstörungen klagten und deren Daten in dieser Publikation näher betrachtet werden. Nach einer Aufnahmeuntersuchung erfolgte nach 90 Tagen eine Abschlussuntersuchung und bei Fortführung der Therapie nach nochmals 90 Tagen eine Follow-up-Untersuchung. Neben 30 ärztlicherseits bewerteten Symptomen beurteilten die Patienten ihr Befinden mittels Pittsburgh Sleep Quality Index (PSQI) nach Buysse und der Beschwerden-Liste nach von Zerssen (B-L und B-L'). Ergebnisse: Unter der Cardiodoron ${ }^{\circledR}$ Therapie gingen bei guter Verträglichkeit sowohl die Ausprägung der Schlafstörungen (um $65 \%$ von 2,0 auf 0,7 Punkte) als auch die erfassten 30 Symptome (um 59\% von 24,3 auf 9,9 Punkte) deutlich zurück $(p<0,01)$. Weiterhin reduzierten sich der PSQI und der Gesamtwert der Beschwerden-Liste signifikant ( $p<0,0001$ ) um $60 \%$ bzw. 56\% (von 12,2 auf 4,8 bzw. von 25,6 auf 11,4 Punkte). Schlussfolgerungen: Bei Patienten mit Schlafstörungen (mit oder ohne funktionelle Herz-Kreislauf-Beschwerden) kann die Ausprägung der Beschwerden mit Cardiodoron ${ }^{\circledR}$ deutlich gesenkt werden. Cardiodoron ${ }^{\circledR}$ ist gut verträglich.

(c) 2016 The Author(s). Published by S. Karger GmbH, Freiburg

\section{Keywords}

Sleep disorders . Prospective, multicenter observational study . Primula. Hyoscyamus . Onopordum

\section{Summary}

Cardiodoron ${ }^{\circledR}$ in Patients with Sleep Disorders - Results of a Prospective Observational Study

Background: Sleep disorders belong to today's most common health problems. Stress, the resulting inner tension, and an arrhythmic life style (e.g. due to shift work) are known to be causative factors. It is less well known that functional cardiovascular disorders can lead to sleep disturbances and that their treatment contributes to improved sleep quality. Therefore, the restoration of healthy functional rhythms, in particular of the cardiac/respiratory rhythm and the sleep-wake rhythm, is the primary aim targeted by Cardiodoron ${ }^{\circledR}$, a composition of 3 medicinal plants: Primula veris, Hyoscyamus niger, and Onopordum acanthium. Patients and Methods: By means of a prospective, multicenter, non-interventional study, the clinical course of functional cardiovascular disorders and/or sleep disorders was evaluated during 3-6 months of treatment with Cardiodoron ${ }^{\circledR}$ (drops). Between September 2009 and March 2012, 92 physicians documented 501 patients, of which 380 complained of insomnia, and their data will be considered in more detail in this publication. 90 days after baseline, a final examination and, in case of continuation of therapy, a follow-up examination after a further 90 days were carried out. Besides 30 symptoms assessed by the physicians, the patients rated their condition on the basis of the Pittsburgh Sleep Quality Index (PSOI) according to Buysse and the complaints list according to von Zerssen (B-L and B-L'). Results: During Cardiodoron ${ }^{\circledR}$ therapy, both the severity of the sleep disturbances (by $65 \%$ from 2.0 to 0.7 points) as well as the documented 30 symptoms (by $59 \%$ from 24.3 to 9.9 points) were significantly reduced ( $p<0.01)$, while Cardiodoron ${ }^{\circledR}$ was well tolerated. Furthermore, the PSOl and the total value of the complaints list were significantly $(p<0.0001)$ reduced by $60 \%$ and $56 \%$, respectively (from 12.2 to 4.8 and from 25.6 to 11.4 points, respectively). Conclusions: In patients with sleep disorders (with or without functional cardiovascular disorders), the severity of complaints can be significantly reduced by Cardiodoron ${ }^{\circledR}$. Cardiodoron ${ }^{\circledR}$ is a well-tolerated medicinal product.

\section{KARGER}

Fax +497614520714 Information@Karger.com www.karger.com

$\begin{array}{lc}\text { (c) } 2016 \text { The Author(s) } & \text { Karger } \\ \text { Published by S. Karger GmbH, Freiburg } & \text { Open access }\end{array}$

This article is licensed under the Creative Commons AttributionNonCommercial-NoDerivatives 4.0 International License (CC BY-NCND 4.0) (http://www.karger.com/Services/OpenAccessLicense). Usage r commercial purposes as well as any distribution of modified material requires written poses as well
Dr. rer. nat. Claudia Rother

WELEDA AG

Möhlerstraße 3, 73525 Schwäbisch Gmünd, Deutschland

crother@weleda.de 


\section{Einleitung}

Cardiodoron ${ }^{\circledR}$ enthält Auszüge aus Primula veris (Schlüsselblume), Hyoscyamus niger (Bilsenkraut) und Onopordum acanthium (Eselsdistel) und gilt als eines der wichtigsten Arzneimittel der Anthroposophischen Medizin [1,2]. Die heute in Deutschland zugelassenen Anwendungsgebiete sind: «Störungen vegetativer Rhythmen und ihrer Koordination, vor allem Herzrhythmusstörungen; Schlafstörungen; Dyskardien und orthostatische Dysregulationen sowie funktionelle Herz- und Kreislaufstörungen bei und nach Infektionskrankheiten» [3]. Die Wirkweise von Cardiodoron ${ }^{\circledR}$ kann derzeit nur eingeschränkt einzelnen pflanzlichen Inhaltsstoffen zugeordnet werden. Bei Primula veris gelten die Saponine als wesentlich. Diese Substanzen wirken seifenähnlich und haben so - qualitativ betrachtet - einen engen Bezug zum «Rhythmusorgan» Lunge bzw. zu den Lungenbläschen, in denen sich ebenfalls «seifenähnlich» - Flüssiges und Luftiges vermischen [46]. Für Primelsaponine ist bekannt, dass sie im Darm nicht resorbiert werden, sondern eine reaktive Antwort im Atemtrakt hervorrufen: eine Schleimlösung und Reinigung der Schleimhäute [7]. Wie genau die Primelinhaltsstoffe im Organismus allerdings wirksam sind, bedarf im Detail noch der Klärung. Für die größte Distel (Onopordum acanthium) wird beschrieben, dass sie durch Stärkung der Stoffwechselprozesse am Herzmuskel auf die Herzfunktion wirkt [4-6]. Neueste Untersuchungen [8] ergaben einen Nachweis ACE-hemmender Inhaltsstoffe im Samen, sodass über diesen Mechanismus der Gefäßtonus gesenkt und der Blutdruck reguliert werden kann. Hyoscyamus niger ist hinsichtlich der ebenfalls Herz-Kreislauf-aktiven Inhaltsstoffe der am besten untersuchte Bestandteil von Cardiodoron ${ }^{\circledR}$. Die enthaltenen Alkaloide Hyoscyamin und Scopolamin weisen schon in kleinen Mengen antitachykarde und antihypertensive Eigenschaften auf. Scopolamin, nah verwandt mit Atropin, wird auch zur Relaxation der glatten Muskulatur eingesetzt [9]. In einem präklinischen Versuch wurde festgestellt, dass der krampflösende Effekt durch eine anticholinerge und $\mathrm{Ca}^{2+}$-antagonistische Wirkung zustande kommt [10]. Untersuchungen am Tier bestätigten die krampflösende und zudem schmerzstillende Wirkung [11-13]. Durch eine transdermale Gabe von Scopolamin konnte eine vagomimetische, blutdrucksenkende und herzfrequenzverlangsamende Wirkung beim Menschen gezeigt werden $[14,15]$. Es kann also insgesamt gefolgert werden, dass Cardiodoron ${ }^{\circledR}$ die Herz-Kreislauf-Regulation im Sinne einer Beruhigung und verbesserten Reagibilität positiv beeinflusst. Auch während des Schlafs finden viele Abstimmungen rhythmischer Funktionen im Herz-Kreislauf-Bereich statt [16]. Diese sind ein Kennzeichen für die Regenerationsleistungen des Organismus. Störungen des Schlafs zeichnen sich unter anderem durch eine verminderte Abstimmung dieser Herz-Kreislauf-Funktionen aus. Cardiodoron ${ }^{\circledR}$ kann die Reagibilität des Herz-Kreislauf-Systems, insbesondere von Herzfrequenz und Blutdruck, günstig beeinflussen, indem im Schlaf vor allem das Verhältnis von Herz- und Atemfrequenz besser aufeinander abstimmt wird [17]. Dies wiederum wirkt sich positiv auf den Schlaf und die subjektive Schlafqualität aus. Prinzipiell unterliegen alle biologischen Pro- zesse im menschlichen Organismus einem zirkadianen Rhythmus und sind eng mit dem Wachen und Schlafen und/oder Hell-Dunkel-Wechseln gekoppelt. Ein besonderer Zusammenhang besteht hinsichtlich der Sekretion von Hormonen, der Körpertemperatur und des Blutdrucks. Vor allem der Hell-Dunkel-Wechsel greift über den Nucleus suprachiasmaticus in diese Rhythmik ein, sodass in den Industrienationen mit den künstlichen Beleuchtungssituationen bereits ein ungünstiger Einfluss vorhanden ist [18]. In der Anthroposophischen Medizin werden dem sogenannten «Rhythmischen System» der Herzrhythmus, der damit eng verknüpfte Atemrhythmus sowie der Schlaf-Wach-Rhythmus zugeordnet. Bei dieser Betrachtungsweise wird klar, dass Schlafstörungen und funktionelle Herz-Kreislauf-Störungen häufig überlappen [5, 19-21].

Die Ursachen von Schlafstörungen sind vielfältig und reichen von einer durch äußere Umstände hervorgerufenen Überreizung im Nerven-Sinnes-Bereich bis hin zu altersbedingten Veränderungen der Schlafes [20, 22-24]. Zudem kommen Insomnie induzierende Medikamente infrage wie durchblutungsfördernde Mittel (z.B. Dihydroergotamin) und Antihypertensiva (z.B. Betablocker) [24]. Schlafstörungen werden nach der International Classification of Sleep Disorders (ICSD) der Amerikanischen Akademie für Schlafmedizin (AASM, American Academy of Sleep Medicine) eingeteilt in: schlafbezogene Atmungsstörungen, Insomnie, Bewegungsstörungen im Schlaf, Hypersomnien, Parasomnien und zirkadiane Schlaf-Wach-Rhythmusstörungen [25]. In Abhängigkeit von der Ursache sind somit ganz unterschiedliche Maßnahmen zu ergreifen, um die jeweilige Schlafstörung zu behandeln. Sofern Störungen des Rhythmischen Systems eine Rolle spielen, ist Cardiodoron $^{\circledR}$ eine mögliche Therapieoption.

Für Cardiodoron ${ }^{\circledR}$ existieren neben einigen Studien mit Patienten [26-31] und mit gesunden Probanden [17, 32] zahlreiche Einzelfallberichte aus der ärztlichen Praxis [33-40]. Vor allem die Kasuistik der niedergelassenen Ärztin Elke Sandra Großklaus stellt anschaulich dar, wie Schlafstörungen und funktionelle Erkrankungen des Herz-Kreislauf-Systems sich gegenseitig bedingen und durch die Einnahme von Cardiodoron ${ }^{\circledR}$ positiv beeinflussen lassen [41]. Bisher lagen jedoch keine systematischen, prospektiven Untersuchungen zur Wirksamkeit und Verträglichkeit von Cardiodoron ${ }^{\circledR}$ hinsichtlich der Anwendung bei Schlafstörungen mit oder ohne funktionelle Herz-Kreislauf-Beschwerden vor. Aufgrund der Häufigkeit dieser Erkrankungen sollte daher im Rahmen einer Beobachtungsstudie unter anderem dokumentiert werden, wie sich Schlafstörungen durch die Behandlung mit Cardiodoron ${ }^{\circledR}$ verändern und welche Arten von Störungen des Schlafs vorliegen sowie der Einfluss außergewöhnlicher Arbeitszeiten.

\section{Patienten und Methoden}

Geplant wurde eine prospektive, nichtinterventionelle, multizentrische Studie mit Cardiodoron ${ }^{\circledR}$ bei 500 auswertbaren Patienten mit funktionellen HerzKreislauf-Beschwerden und/oder Schlafstörungen in ärztlichen Praxen Deutschlands. Die Daten der 380 Patienten mit Schlafstörungen (mit und ohne gleichzeitige Herz-Kreislauf-Beschwerden) stehen im Fokus dieser Publikation. 


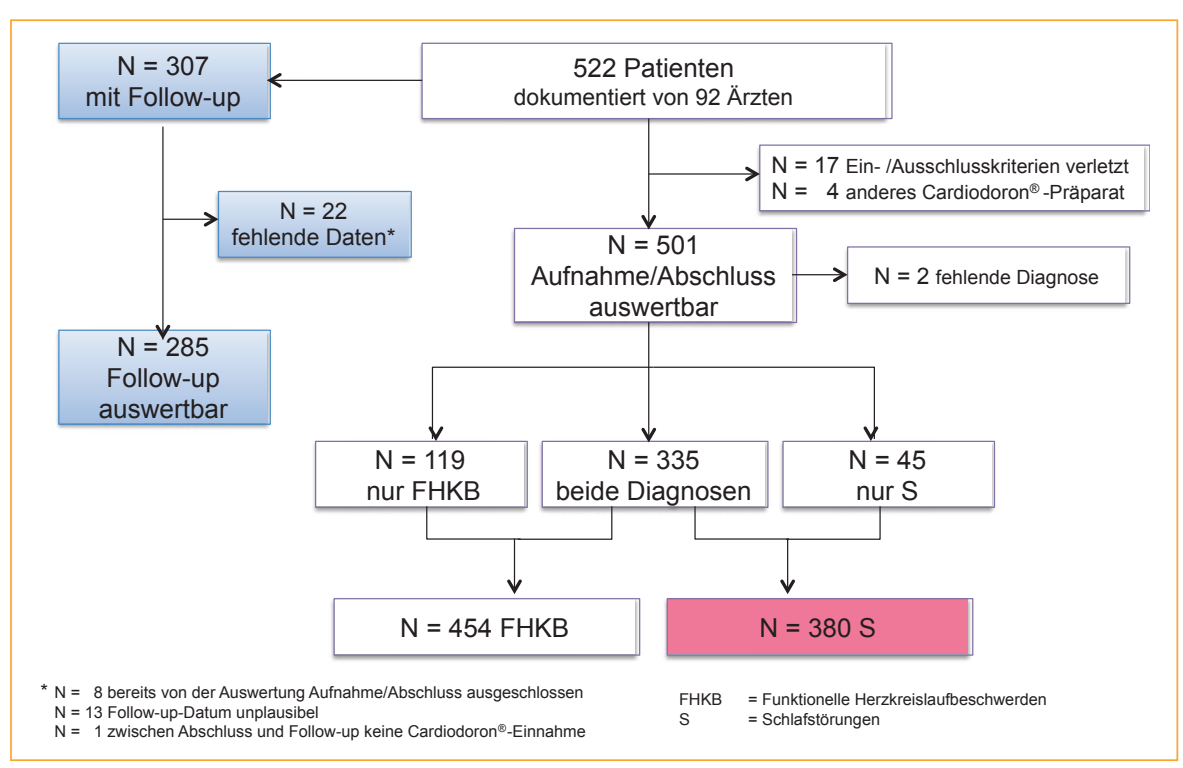

Abb. 1. Patientenkollektiv und Diagnosen.

Nach einer Aufnahmeuntersuchung nahmen die Patienten über $90( \pm 10)$ Tage Cardiodoron ${ }^{\circledR}$-Tropfen ein und erschienen dann zu einer Abschlussuntersuchung. Sofern Cardiodoron ${ }^{\circledR}$ weiter verordnet wurde, konnte nach nochmals $90( \pm 10)$ Tagen ein Follow-up dokumentiert werden. Im Wesentlichen erfolgte ärztlicherseits die Erfassung der Ausprägung der Schlafstörungen sowie die Bewertung von 30 krankheitstypischen Symptomen mit 0 Punkten $=$ nicht vorhanden bis 3 Punkten $=$ schwer ausgeprägt. Diese 30 Symptome wurden in Hinsicht auf die behandelten Indikationen studienspezifisch ausgewählt. Begleiterkrankungen und -therapien erfasste der behandelnde Arzt fortlaufend bis zur Abschlussuntersuchung. Die Cardiodoron ${ }^{\circledR}$-Dosierung und -Compliance, die Dauer bis zum Wirkungseintritt sowie die Beurteilung der Wirksamkeit und Verträglichkeit wurden ebenfalls erhoben. Bei jeder Untersuchung füllten die Patienten die Beschwerden-Liste B-L + B-L' nach von Zerssen [42] aus. Dieses Selbsteinschätzungsinstrument wurde zur quantitativen Bewertung der subjektiven Beeinträchtigung durch körperliche und allgemeine Beschwerden entwickelt. Folgende Bewertung der Beschwerden kam zur Anwendung: stark = 3 Punkte, mäßig $=2$ Punkte, kaum $=1$ Punkt, gar nicht $=0$ Punkte. Zur Interpretation der Ergebnisse dienten die Normwerte im Anhang $\mathrm{C}$ des Manuals. Für den Gesamtwert beider Listen, berechnet als $\left(\mathrm{B}-\mathrm{L}+\mathrm{B}-\mathrm{L}^{\prime}\right) / 2$, gelten folgende Werte für das Gesamtkollektiv als kritisch: 22-27 = fraglich abnorm, $\geq 28=$ sicher abnorm [42]. Der Fragebogen zur Schlafqualität nach Buysse (Pittsburgh Sleep Quality Index (PSQI)) [43] stellt mit 18 Selbstbeurteilungsfragen und 5 Fragen, die vom Partner/Mitbewohner beurteilt werden, ein einfaches und effektives Verfahren zur Erfassung der subjektiven Schlafqualität dar. International findet der Bogen vor allem bei Untersuchungen von Populationen außerhalb des Schlaflabors Verwendung und ist für wiederholte Messungen gut geeignet [44]. Riemann und Backhaus [44] übertrugen den PSQI ins Deutsche und verkürzten die rückschauende Einschätzung des Schlafes von 4 Wochen auf für den Patienten besser beurteilbare 14 Tage. Es erschien sinnvoll, dieses Selbsteinschätzungsinstrument bei Patienten mit Schlafstörungen analog zu Riemann und Backhaus bei jedem Untersuchungszeitpunkt einzusetzen. In die Auswertung des PSQI-Gesamtwertes gingen nur die 18 Selbstbeurteilungsfragen ein, die zu den 7 Komponenten Schlafqualität, Schlaflatenz, Schlafdauer, Schlafeffizienz, Schlafstörungen, Schlafmittelkonsum und Tagesschläfrigkeit zusammengefasst wurden. Jede Komponente kann einen Wert zwischen 0 und 3 Punkten annehmen, wobei 0 für «keine Schwierigkeiten» und 3 für «große Schwierigkeiten» steht. Der PSQI-Gesamtwert ergibt sich aus den 7 addierten Komponentenwerten mit einem Wert zwischen 0 und 21. Ein Gesamtwert von mehr als 5 Punkten gilt als auffällig und weist auf eine schlechte Schlafqualität hin [44]. Die Antworten auf die 5 Fragen an den Mitbewohner/Partner dienen qualitativ als Hinweise für das Vorliegen organischer Faktoren wie z.B. Restless Legs. In diese deskriptive Auswertung gingen nur die Patienten ein, die nicht allein geschlafen haben und bei denen eine weitere Person die Schlafqualität beurteilen konnte.

Die Details zur deskriptiven Auswertung wurden vor Beginn der Datenanalyse mittels eines statistischen Analyseplans festgelegt. Analog erfolgte die Festlegung des auswertbaren Patientenkollektivs. Die Auswertung der erhobenen Parameter war deskriptiv bzw. explorativ geplant. Die Darstellung kontinuierlicher Merkmale erfolgte mit: Anzahl der Beobachtungen, Mittelwert, Standardabweichung, Minimum, Median und Maximum. Kategorielle Merkmale wurden mit absoluten und relativen Häufigkeiten innerhalb der einzelnen Kategorien abgebildet. Subgruppenanalysen waren in Abhängigkeit von der Datenlage vorgesehen und ergaben sich aus demografischen, anamnestischen sowie klinischen bzw. untersuchungsspezifischen Kriterien oder deren Kombinationen. Sofern die Berechnung von p-Werten für Unterschiede zwischen Subgruppen sinnvoll erschien, wurden diese mittels t-Test, Mann-Whitney U-Test und Wilcoxon-Test ohne Bezug auf Hypothesen aufgeführt. Generell fand keine Fehleradjustierung bezüglich des multiplen Testens statt. Die verwendeten Softwares für die Auswertung waren SPSS Version 19 sowie R-2.11.1 und Statistica 10.

\section{Ergebnisse}

\section{Patientenkollektiv, Diagnosen und demografische Daten}

Von September 2009 bis März 2012 dokumentierten 92 Ärzte insgesamt 522 Patienten, von denen 501 in die Auswertung eingingen und deren Daten in einem Übersichtsartikel bereits publiziert wurden [45]. Der größte Teil aller Patienten, d.h. 67\% ( $\mathrm{N}=335)$, litt sowohl unter funktionellen Herz-Kreislauf-Beschwerden als auch unter Schlafstörungen. Der Anteil der Patienten, die ausschließlich über Schlafstörungen klagten, lag bei $9 \%$ bzw. N = 45 (Abb. 1). Die insgesamt 380 Patienten mit Schlafstörungen stehen im Fokus dieser Publikation.

Rund 80\% ( $\mathrm{N}=297)$ der Patienten mit Schlafstörungen gaben an, Durchschlafschwierigkeiten zu haben, gefolgt von $62 \%(\mathrm{~N}=$ 234) mit Einschlafproblemen und $45 \%(\mathrm{~N}=170)$ mit vorzeitigem Erwachen (Mehrfachnennungen möglich). Bei der Angabe der Gründe für diese Beschwerden wurde von 178 Patienten der Freitext genutzt. Vor allem Stress, Belastungen in Job und Familie sowie Unruhe/Ängste führten zu einer unzureichenden Schlafqua- 
Abb. 2. Ausprägung der Schlafstörungen.

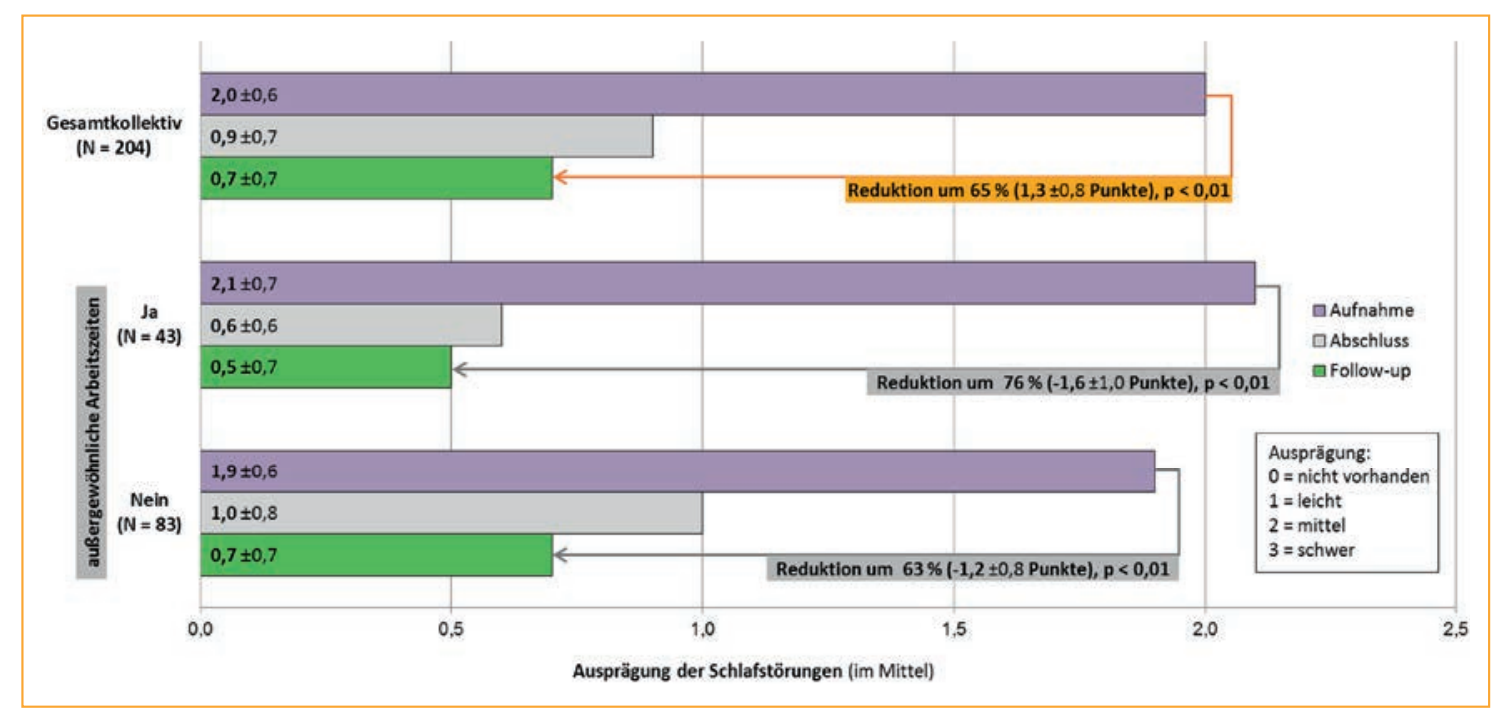

lität. Weiterhin trafen die direkt anzukreuzenden Gründe - Jetlag und Schichtarbeit - bei 31 bzw. 22 Patienten zu. Im Mittel bestanden die Schlafstörungen bereits seit über 3 Jahren (39 Monate). Für rund die Hälfte der Patienten ( $\mathrm{N}=207$ ) wurden sehr unterschiedliche weitere Begleiterkrankungen dokumentiert. Im Mittel lag das Alter der Patienten bei 53 Jahren. Weibliche Patienten waren mit $73 \%(\mathrm{~N}=279)$ deutlich häufiger vertreten als männliche $(27 \%, \mathrm{~N}=$ 101). Knapp über die Hälfte aller Patienten war berufstätig ( $\mathrm{N}=$ 199); außergewöhnliche Arbeitszeiten lagen bei 74 der 380 Patienten vor.

\section{Therapie}

Gemäß Fachinformation wird 1- bis 3-mal täglich folgende Cardiodoron ${ }^{\circledR}$-Einnahme empfohlen: Erwachsene und Jugendliche ab 12 Jahren: 15-20 Tropfen; Schulkinder von 6 bis 11 Jahren: 8-10 Tropfen; Kleinkinder bis zu 5 Jahren: 3-8 Tropfen. Der größte Anteil der Patienten, d.h. 67\% ( $\mathrm{N}=255)$, erhielt die empfohlene Dosierung, gefolgt von einer niedrigeren Dosierung bei $28 \%(\mathrm{~N}=108)$. Hinsichtlich der empfohlenen Tagesdosis (15-60 Tropfen pro Tag bei Erwachsenen und Jugendlichen) lagen 360 bzw. 95\% der Patienten mit Schlafstörungen innerhalb dieses Bereichs. Die am häufigsten angewendeten Dosierungen waren 3bzw. 2-mal täglich 15-20 Tropfen ( $\mathrm{N}=147$ bzw. $\mathrm{N}=71)$. Die Compliance wurde zu 67\% mit «sehr gut» und zu 24\% mit «gut» bewertet.

Über die Hälfte der Patienten mit Schlafstörungen ( $\mathrm{N}=220)$ erhielt weitere Begleittherapien. Diese waren schwerpunktmäßig Herz-Kreislauf-Mittel und sonstige Therapien. Lediglich 15 Schlafmittel wurden genannt; 9 davon waren klassische allopathische Präparate. Neben dieser Dokumentation durch den Arzt wurden die Patienten im PSQI direkt gefragt, ob sie in den letzten 2 Wochen Schlafmittel genommen hatten und, wenn ja, welche. Bei der Abschlussuntersuchung gaben 308 der 380 Patienten an, keine Schlafmittel genommen zu haben, 33 nahmen Schlafmittel, wobei 24 Präparate genannt wurden. Klassische allopathische Schlafmittel waren auch hier nur vereinzelt darunter (15 Nennungen).
Von den insgesamt 380 Patienten wurden 99 Patienten (26,1\%) bezüglich ihrer Schlafstörungen bereits früher medikamentös behandelt (insgesamt 118 Nennungen, Mehrfachnennungen pro Patient möglich, bezeichnet als Vormedikation). Hypnotika und Sedativa erhielten 65 Patienten (d.h. 55\% bezogen auf 118 Nennungen), gefolgt von 18 Patienten (15\%) mit Antidepressiva, 14 Patienten (12\%) mit Homöopathika und Anthroposophika und 9 Patienten (8\%) mit Anxiolytika.

\section{Wirksamkeit}

Die Ausprägung der Schlafstörungen reduzierte sich im Behandlungsverlauf signifikant (jeweils $\mathrm{p}<0,01$ ), und zwar von 2,0 auf 0,9 bei Abschluss und 0,7 bei Follow-up, d.h. die anfänglich «mittelstark» ausgeprägten Schlafstörungen waren bei Behandlungsende nur noch «leicht» vorhanden. Deutlicher zeigte sich dieser Rückgang bei Patienten mit außergewöhnlichen Arbeitszeiten. Die Patienten mit vollständigen Werten an den 3 Untersuchungszeitpunkten $(\mathrm{N}=43)$ wiesen zu Beginn einen Wert von 2,1 auf, der sich bereits nach 3 Monaten Cardiodoron ${ }^{\circledR}$-Einnahme auf 0,6 und nach 6 Monaten auf 0,5 reduzierte. Dies entspricht einer deutlichen Reduktion ( $\mathrm{p}<0,01$ ) von 1,6 Punkten bzw. 76\% (Abb. 2). Verglichen mit Patienten ohne außergewöhnliche Arbeitszeiten $(\mathrm{N}=83)$ profitierten diese Patienten signifikant stärker $(\mathrm{p}<0,02$ bezüglich des Vergleichs der Differenzen Aufnahme/Follow-up zwischen den Gruppen).

Der Schweregrad aller 30 erfassten Symptome verminderte sich durchgehend zwischen Aufnahme- und Abschlussuntersuchung. Eine weitere Verbesserung konnte bei der Follow-up-Untersuchung bei fast allen der in Abbildung 3 im Detail dargestellten Symptome beobachtet werden. Aus diesen 30 Symptomen wurde der Symptom-Summenscore errechnet. Es konnte ein jeweils signifikanter Rückgang des Symptom-Summenscores $(\mathrm{p}<0,01)$ zwischen Aufnahme und Abschluss bzw. Follow-up ermittelt werden. In Punkten bedeutet dies: 24,3 versus 12,1 Punkte versus 9,9. Auch bezüglich des Symptom-Summenscores schienen Patienten mit außergewöhnlichen Arbeitszeiten besonders zu profitieren. Die Re- 


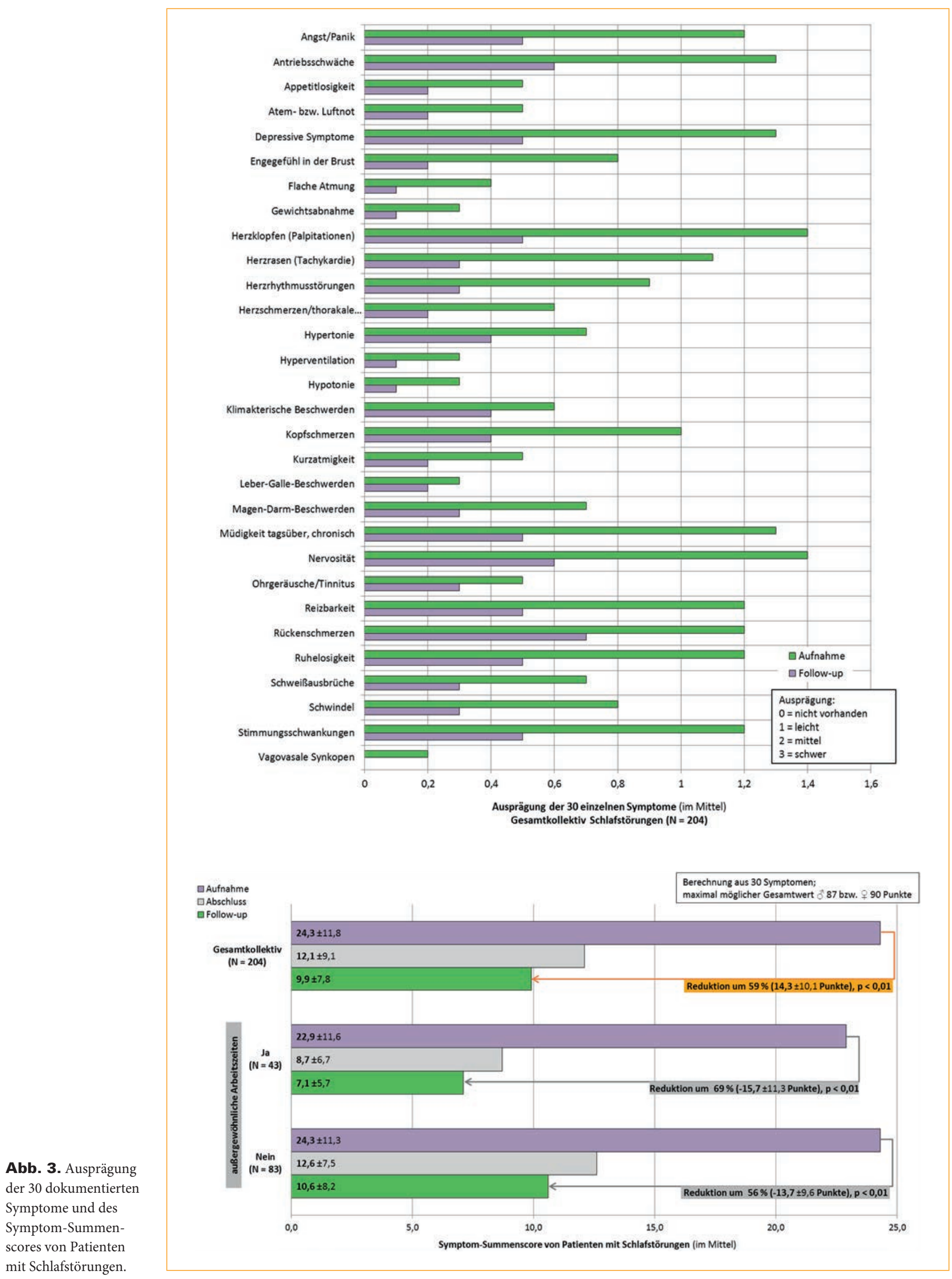




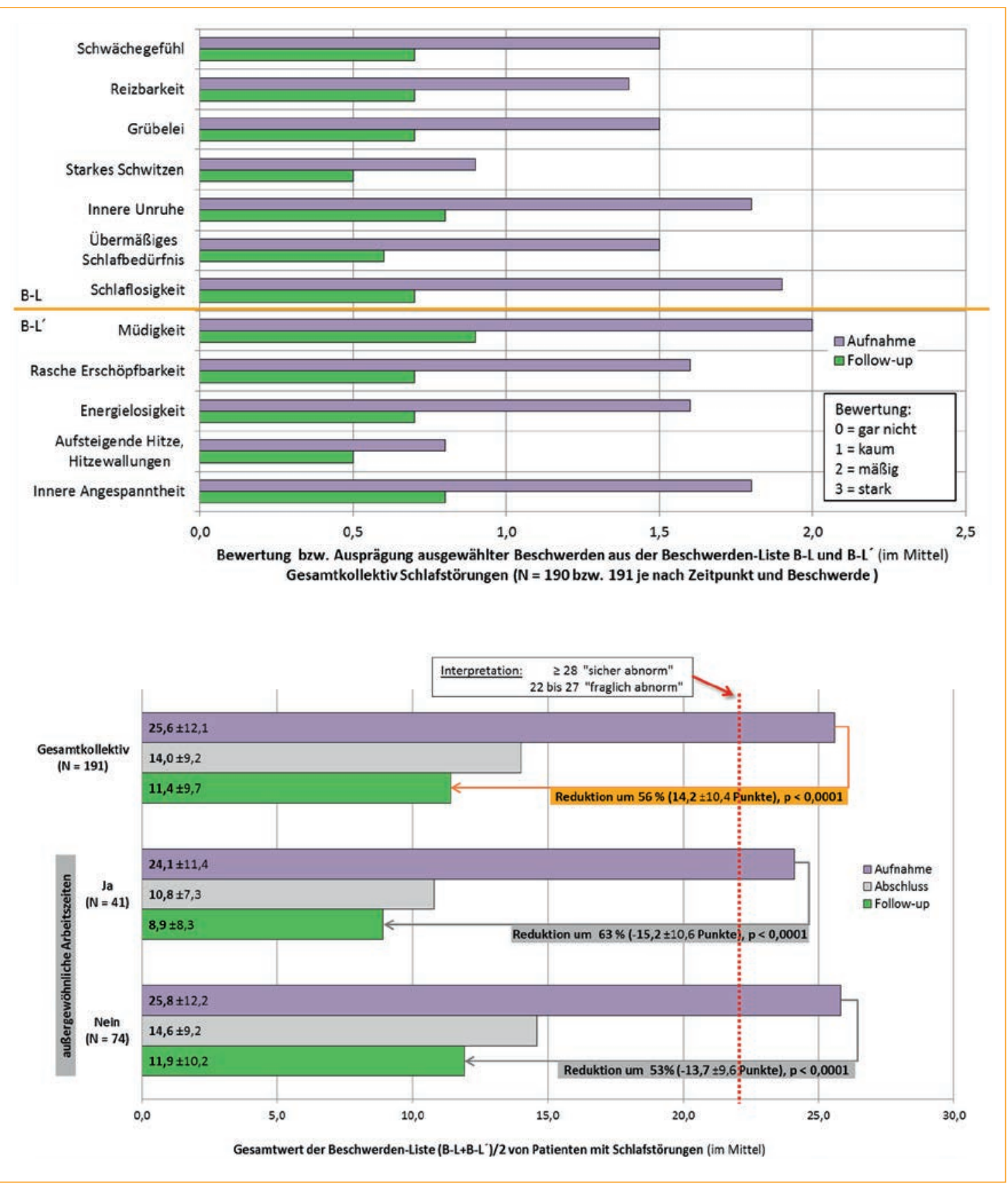

Abb. 4. Ausprägung einzelner Beschwerden der Beschwerden-Liste (B-L/B-L') und Gesamtwert $\left(\mathrm{B}-\mathrm{L}+\mathrm{B}-\mathrm{L}^{\prime}\right) / 2$ von Patienten mit Schlafstörungen.

duktion betrug 15,7 Punkte (-69\%, p < 0,01) im Vergleich zu Patienten ohne diese berufliche Anforderung mit 13,7 Punkten (-56\%, $\mathrm{p}<0,01$ ) (Abb. 3), wobei der Unterschied zwischen den Gruppen statistisch nicht gesichert werden konnte.

Die Ausprägung jeder Beschwerde der Beschwerden-Liste nach von Zerssen [42] war zwischen Aufnahme, Abschluss und Followup rückläufig. Abbildung 4 stellt eine Auswahl der für die Indikation Schlafstörungen besonders relevanten Beschwerden aus den Teillisten B-L und B-L' dar sowie den Gesamtwert aller Beschwerden, berechnet als $\left(B-L+B-L^{\prime}\right) / 2$. Letzterer lag zu Behandlungsbeginn mit 25,6 Punkten in einem kritischen Bereich, der gemäß den Normwerten als «fraglich abnorm» definiert wird. Bei Abschluss bzw. zum Follow-up konnten 14,0 bzw. 11,4 Gesamtpunkte ermittelt und somit der Normalbereich erreicht werden. Die Daten der
Patienten mit außergewöhnlichen Arbeitszeiten ergaben auch bezüglich der Beschwerden-Liste tendenziell einen Vorteil gegenüber Patienten ohne diese Einschränkung. Die Reduktion der Beschwerden lag bei 63\% versus 53\% (Abb. 4), wobei der Gruppenunterschied statistisch nicht signifikant war.

Der Fragebogen zur Schlafqualität (PSQI) nach Buysse [43] zeigte ähnliche Verbesserungen (Abb. 5). Insgesamt 6 der 7 Einzelkomponenten - d.h. Schlafqualität, Schlaflatenz, Schlafdauer, Schlafeffizienz, Schlafstörungen und Tagesmüdigkeit - lagen zu Behandlungsbeginn zwischen 2,7 und 1,3 Punkten, d.h. in einem kritischen Bereich. Insbesondere hatten die Patienten hinsichtlich der Schlaflatenz, d.h. mit der Zeit bis zum Einschlafen, die größten Schwierigkeiten. Nach 3-monatiger Behandlung reduzierten sich die Werte durchgehend um rund die Hälfte. Die Komponente «Schlafmittel- 


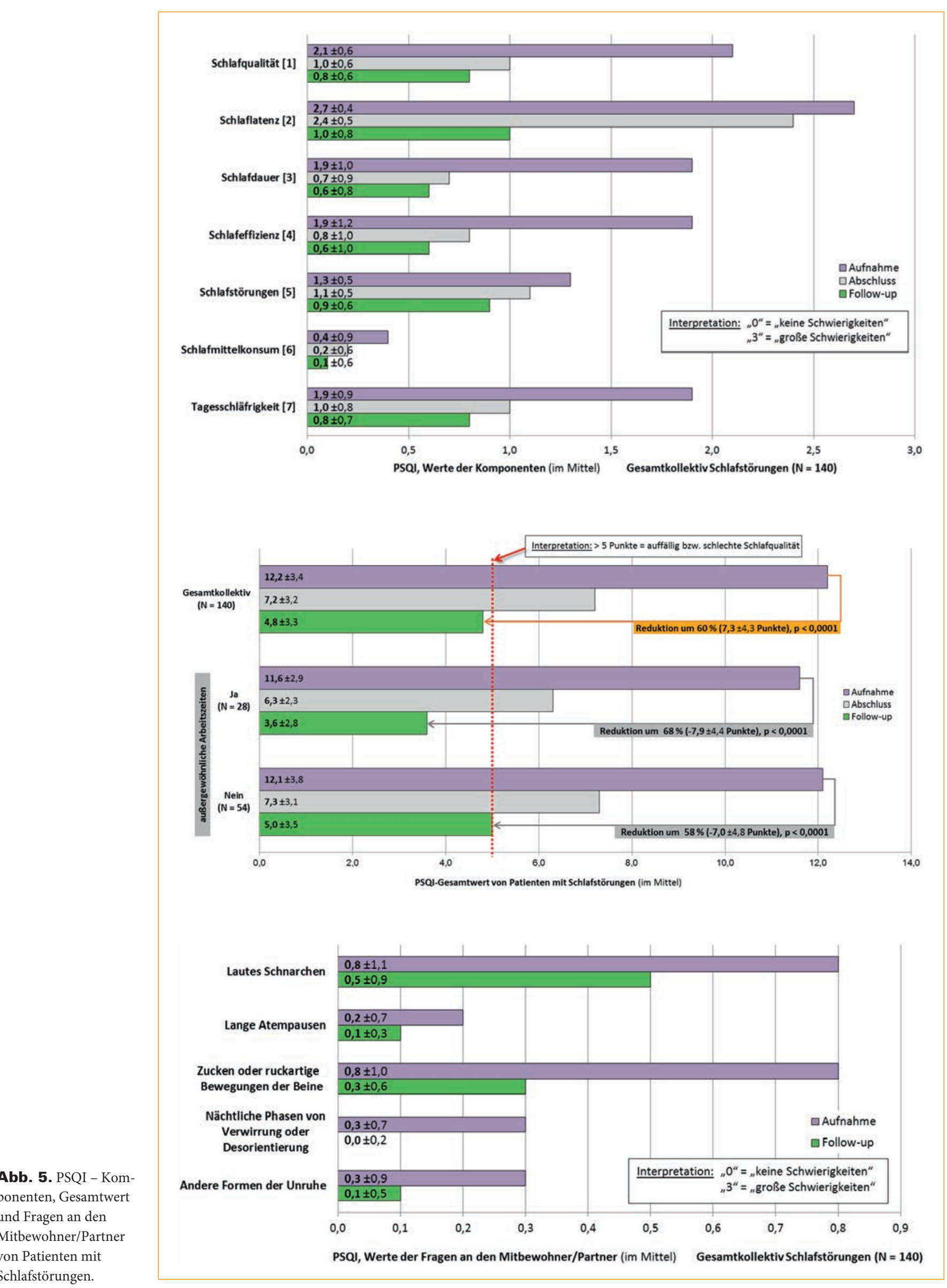


Abb. 6. Wirksamkeits- und Verträglichkeitsbewertung (Arzt) von Cardiodoron ${ }^{\circledR}$ versus Vormedikation von Patienten mit Schlafstörungen bzw. im Gesamtkollektiv.

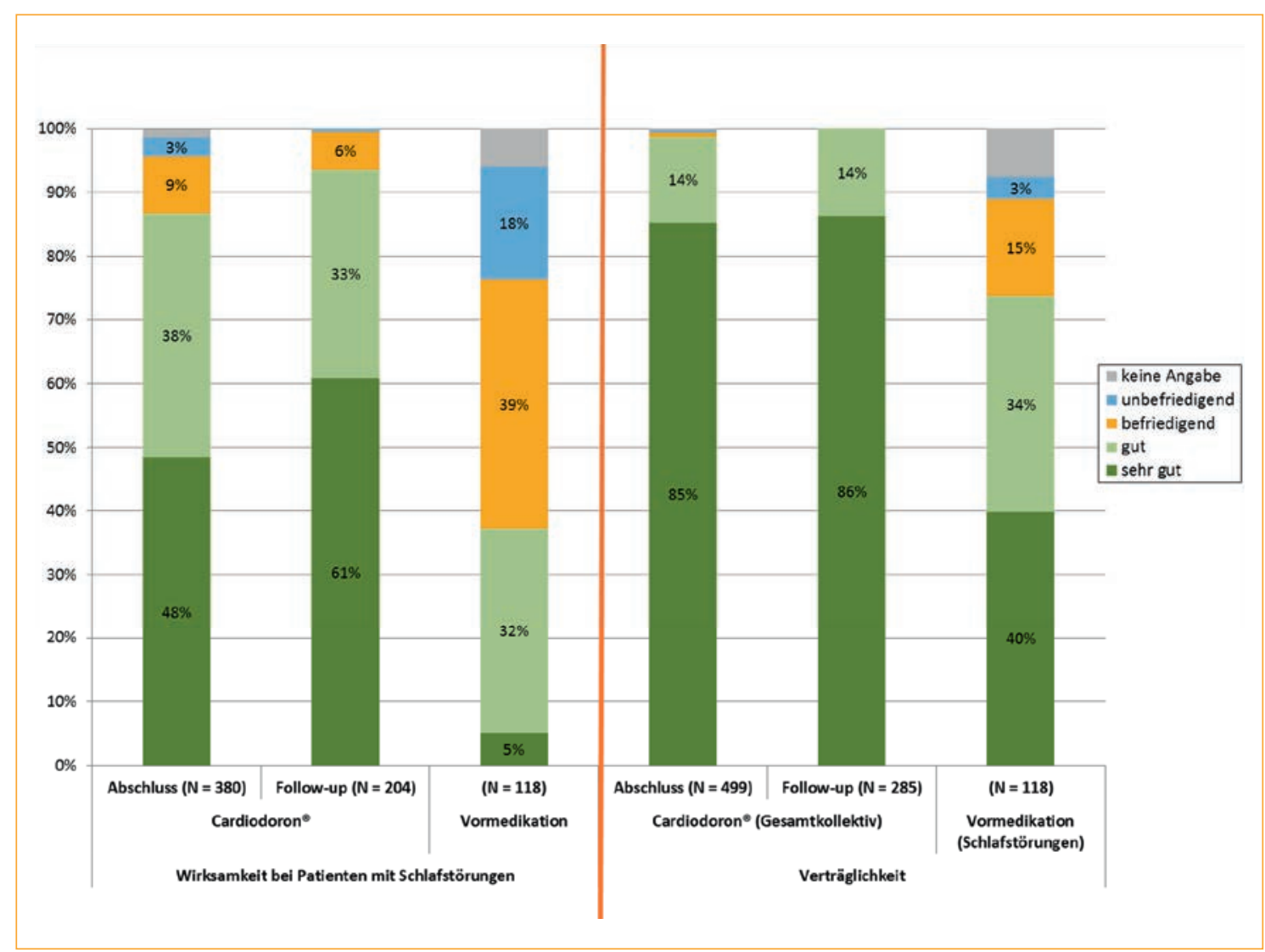

konsum» wies bei Aufnahme im Mittel mit 0,4 Punkten einen eher geringen Wert auf; auch dieser konnte auf 0,2 bzw. 0,1 Punkte gesenkt werden. Die Schlafdauer, die in Deutschland bei durchschnittlich 7,25 h liegt [18], befand sich anfänglich mit 1,9 Punkten in einem Bereich zwischen 5 und 6 h. Mit 0,6 Punkten zum Followup erreichte die Schlafdauer dann im Mittel über 7 h. Der PSQIGesamtwert lag zu Beginn im Mittel bei 12,2 Punkten und reduzierte sich nach 3-monatiger Cardiodoron ${ }^{\circledR}$-Einnahme auf 7,2 und nach weiteren 3 Monaten zum Follow-up auf 4,8 Punkte. Prozentual betrachtet bedeutet dies einen Rückgang der Schlafprobleme um $60 \%$. Weiterhin ist ersichtlich, dass sich nach 3-monatiger Behandlung mit Cardiodoron ${ }^{\circledR}$ bei Patienten mit außergewöhnlichen Arbeitszeiten der PSQI-Gesamtwert um 7,9 Punkte $(\mathrm{N}=28)$ im Vergleich zu Patienten ohne diese Einflussgröße mit 7,0 Punkten $(\mathrm{N}=54)$ reduzierte. Der Unterschied zwischen den Gruppen lag nicht im statistisch signifikanten Bereich. Auch die Antworten auf die Fragen an den Mitbewohner/Partner als Hinweise für das Vorliegen organischer Faktoren wie z.B. Restless Legs ergaben Verbesserungen, wie Abbildung $5 \mathrm{zu}$ entnehmen ist.

Insgesamt gesehen stellten $92 \%$ der Patienten eine Wirksamkeit der Cardiodoron ${ }^{\circledR}$-Dilution fest, d.h. 349 der 380 Patienten. Ein erster Eintritt der Wirksamkeit wurde nach 13 Tagen ( \pm 10 Tage) dokumentiert. Auch Patienten, die ausschließlich über Schlafstörungen klagten, bescheinigten zu 96\% eine Wirkung (43 der $45 \mathrm{~Pa}$ tienten). Bei diesen Patienten dauerte es mit im Mittel 17 Tagen ( \pm 15 Tage) etwas länger, bis eine Wirkung festgestellt werden konnte.
Bei der Beurteilung der Wirksamkeit von Cardiodoron ${ }^{\circledR}$ wurde ärztlicherseits bei Abschluss bzw. Follow-up zu 48\% bzw. zu 61\% «sehr gut» vergeben, gefolgt von «gut» mit 38\% bzw. 33\%. Das heißt, für insgesamt 330 von 380 Patienten (86\%) bestätigten die Ärzte nach 3-monatiger Behandlung Cardiodoron ${ }^{\circledR}$ eine sehr gute bis gute Wirksamkeit. Nach insgesamt 6 Monaten waren es von den bis dahin dokumentierten Patienten $(\mathrm{N}=204)$ in Summe 191, d.h. 94\% (Abb. 6). Ein ähnliches Bild ergab die Bewertung der Wirksamkeit durch die Patienten: Follow-up-Bewertung mit «sehr gut» 54\%, «gut» 35\%. Für die Vormedikation (99 Patienten mit 118 Nennungen) bescheinigten $5 \%$ der Ärzte $(\mathrm{N}=6)$ (Abb. 6) und $4 \%$ der Patienten $(\mathrm{N}=5)$ eine «sehr gute» Wirkung. Weiterhin würden $89 \%$ der Ärzte Cardiodoron ${ }^{\circledR}$ wieder verordnen; nur 2\% verneinten die Frage danach ( $9 \%$ keine Angaben).

\section{Verträglichkeit}

Da die Verträglichkeit der Cardiodoron ${ }^{\circledR}$-Dilution im Gesamtkollektiv von 501 Patienten durch die Ärzte sowie die Patienten bei der Abschlussuntersuchung mit 99\% bzw. 98\% als «sehr gut/gut» beurteilt wurde, fand nicht nochmals eine separate Auswertung für die Patienten mit Schlafstörungen statt (Abb. 6). Die gute Verträglichkeit zeigte sich auch in der geringen Anzahl der Verdachtsfälle von Nebenwirkungen für das Gesamtkollektiv: $1 \times$ Hustenreiz, $2 \times$ Unruhe, $1 \times$ allergische Hautreaktion und $1 \times$ Magenbeschwerden. Für die 99 Patienten, die bezüglich ihrer Schlafstörungen bereits früher medikamentös (insgesamt 118 Nennungen) behandelt wurden, erfolgte eine Bewertung mit «sehr gut/gut» in $74 \%$ der Fälle. 


\section{Diskussion}

Cardiodoron ${ }^{\circledR}$ «hat keine umschriebene Indikation, sondern ein wesentlich umfassenderes Wirkungsspektrum. Es ist nicht alleine ein Mittel bei Herzrhythmusstörungen, bei Hypotonie oder arterieller Hypertonie, bei Herzinfarkt und anderen Erkrankungen des Herzkreislaufsystems: Man kann es ganz allgemein bei Störungen rhythmischer Prozesse anwenden» [46]. Dieses Zitat spiegelt sich in den zugelassenen Anwendungsgebieten wider, die mit «Störungen vegetativer Rhythmen und ihrer Koordination» beginnen sowie «funktionelle Herz-Kreislauf-Störungen» und «Schlafstörungen» einschließen [3]. In der Literatur finden sich für Cardiodoron ${ }^{\circledR}$ vor allem Hinweise zum Einsatz bei Beschwerden des Herz-KreislaufSystems; nur vereinzelt wird darauf hingewiesen, dass auch ein beeinträchtigter Schlaf positiv beeinflusst werden kann [41, 47-49]. Eine Umfrage bei Ärzten ergab bereits in den 1980er-Jahren, dass Cardiodoron ${ }^{\circledR}$ in über $80 \%$ der Fälle bei funktionellen Kreislaufstörungen einsetzt wurde; $12 \%$ der Ärzte berichteten unabhängig von der Primärerkrankung von einem verbesserten Schlaf der Patienten [48]. Schlafstörungen gehören zu den häufigsten Beschwerden der heutigen Zeit (30\% der westlichen Bevölkerung; 20\% bereits bei Jugendlichen/jungen Erwachsenen) und stellen ein nicht unerhebliches Risiko für Folgeerkrankungen dar [23, 50]. Oftmals gehen Erkrankungen des Herz-Kreislauf-Systems mit Schlafstörungen einher $[25,35,51-54]$, sodass in dieser Studie beide Indikationen näher betrachtet wurden. Der Zusammenhang zwischen Schlaf und dem Herzkreislauf steht derzeit generell im Interesse der Forschung. Sowohl im Tiermodell [55] als auch beim Menschen [56] wurde eine erhöhte kardiovaskuläre Sterblichkeit festgestellt, sobald ein stark veränderter Tag-Nacht-Rhythmus vorlag. Weiterhin stehen die durch Schlafmangel am stärksten hochregulierten Gene über eine B-Zellaktivierung sowie eine Erhöhung des C-reaktiven Proteins im direkten Zusammenhang zum Immunsystem [57]. Eine Verbindung zwischen der «inneren Uhr» sowie der Immun- und Stress-Signalwege scheint zudem das Enzym Proteinkinase p38 zu sein. Dunkelheit und Stress beeinflussen dessen Aktivierung [58]. Pillai [21] beobachtete bei einer Gruppe gesunder Probanden eine Erhöhung des Insomnie-Risikos, je mehr Belastungsfaktoren vorlagen und je länger der Stress anhielt.

Auch die Ergebnisse der hier vorgestellten Beobachtungsstudie weisen darauf hin, dass Schlaf- bzw. Herz-Kreislauf-Beschwerden im Zusammenhang stehen: Der größte Teil der Patienten (335 von 501) litt unter beiden Störungen. Funktionelle Erkrankungen kommen bei Frauen bis zu 3-mal häufiger vor als bei Männern [52]. Diese Verteilung bestätigte sich mit einem Frauenanteil von über 70\% in dieser Studie. Das festgestellte Alter von im Mittel 53 Jahren gilt unter anderem aufgrund hoher beruflicher und privater Anforderungen in dieser Lebensphase als charakteristisch [23, 24, 37, 41, 59]. Über die Hälfte der Patienten mit Schlafstörungen (199 von 380) waren berufstätig; davon 74 mit außergewöhnlichen Arbeitszeiten, d.h. 19,5\%. Diese Prozentzahl ist auch in der Literatur zu finden: In der westlichen Arbeitswelt sind 20\% der Beschäftigten in Schichtbetrieb bzw. Nachtarbeit tätig [56]. Daneben gaben die Patienten im Freitext als Gründe für ihre Beschwerden neben
Jetlag und Schichtarbeit vor allem Stress, Belastung in Job und Familie sowie Unruhe und Ängste an. Gemäß Heitmann et al. [60] und Fintelmann [20] sind Ein- und Durchschlafstörungen die häufigsten schlafmedizinischen Störungen. Auch die Patienten dieser Studie litten vorrangig unter Durchschlafschwierigkeiten (80\%), gefolgt von Einschlafproblemen (62\%). Eine funktionelle Erkrankung gilt ab einer Beschwerdedauer von über 6 Monaten als chronisch [52]. Schlafstörungen werden als primäre Insomnie bezeichnet, wenn sie seit 1 Monat vorhanden sind, wenigstens 3-mal pro Woche auftreten und negative Auswirkungen auf die Tagesbefindlichkeit haben [21, 61]. Daher kann für das Patientenkollektiv dieser Studie festgestellt werden, dass die Schlafstörungen bereits chronische Züge angenommen hatten, da die Beschwerden im Mittel bereits 39 Monate vorhanden waren. Auch die sogenannten Vortherapien konnten die Chronifizierung nicht nachdrücklich verhindern. Für 26\% (N = 99) der Patienten lagen dazu Angaben vor; 65 Patienten erhielten gegen ihre Schlafstörungen bereits einmal Medikamente aus der Gruppe der Hypnotika und Sedativa. In diesem Zusammenhang erscheint folgendes Zitat [62] passend: «Man sollte ... immer darauf hinweisen, daß man mit einem Schlafmittel eine Schlaflosigkeit ebenso wenig heilen kann wie eine Karies mit einem Schmerzmittel!» Mit Cardiodoron ${ }^{\circledR}$ hingegen soll die Schlaflosigkeit geheilt und nicht nur symptomatisch behandelt werden, was allerdings im Rahmen dieser Beobachtungsstudie aus methodischen Gründen nicht direkt verifiziert werden konnte. Die einfachste Art, die Wirksamkeit von Cardiodoron ${ }^{\circledR}$ näher zu beleuchten, ist die Bewertung der Ausprägung der Schlafstörungen, die zu Behandlungsbeginn mit 2,0 Punkten mittelstark einzustufen war und sich nach 3 bzw. 6 Monaten Cardiodoron ${ }^{\circledR}$ Einnahme signifikant $(\mathrm{p}<0,01)$ auf leichte Beschwerden mit 0,9 bzw. 0,7 Punkten reduzierte (-65\%). Ferner ist bekannt, dass Menschen mit außergewöhnlichen Arbeitszeiten häufiger über Schlafstörungen klagen $[18,25]$. Somit verwundert es nicht, dass der Schweregrad der Schlafstörungen dieser Patienten mit 2,1 Punkten zu Behandlungsbeginn etwas höher lag. Interessanterweise reduzierte sich der Wert bereits deutlich bei Abschluss auf 0,6 Punkte bzw. zum Follow-up auf 0,5 Punkte (-76\%). Im Vergleich zu Patienten ohne diese berufliche Anforderung profitierten die Patienten zudem signifikant stärker von der Cardiodoron ${ }^{\circledR}$-Einnahme. Das spricht für die in der Einleitung bereits ausführlich diskutierte Wirkrichtung des Präparates.

Die Betrachtung der erfassten 30 Symptome ergab ähnliche Resultate. Alle Symptome verbesserten sich durchgehend, sodass der Symptom-Summenscore von 24,3 Punkten auf 9,9 beim Follow-up sank (-59\%). Die Reduktion des Symptom-Summenscores der Patienten mit versus ohne außergewöhnliche Arbeitszeiten lag mit $-69 \%$ versus $-56 \%$ in einer ähnlichen Größenordnung wie die Ausprägung der Schlafstörung; allerdings ohne statistisch gesicherten Unterschied zwischen den Subgruppen.

Die von den Patienten ausgefüllte Beschwerden-Liste nach von Zerssen untermauert die ärztlicherseits dokumentierten Daten: Der anfänglich kritische Gesamtwert von 25,6 sank auf ein als normal einzustufendes Niveau, d.h. beim Follow-up auf 11,4. Ähnliche Werte wurden in der 2012 publizierten klinischen Prüfung bei Pa- 
tientinnen mit hypotonen Kreislaufstörungen ermittelt [30, 31]. Der Fragebogen zur Schlafqualität PSQI zur Erfassung der subjektiven Qualität des Schlafs weist mit mehr als 5 Punkten auf eine schlechte Schlafqualität hin. Somit litten die Patienten zu Behandlungsbeginn mit einem PSQI-Gesamtwert von 12,2 Punkten deutlich unter Schlafstörungen. Nach 3 bzw. 6 Monaten verminderte sich dieser Wert auf 7,2 bzw. 4,8. Diese Punktezahl liegt damit im unkritischen Bereich und die Verbesserung der Schlafproblematik ist offensichtlich. Allerdings muss beachtet werden, dass der Schlaf im PSQI subjektiv eingeschätzt wurde und diese Einschätzung von objektiv messbaren Werten, wie sie beispielsweise durch eine Polysomnografie erhoben werden, abweichen kann [60]. Für den Patienten ist jedoch in erster Linie die subjektive Empfindung das entscheidende Maß, d.h. die erlebte Erholung, und diese konnte durch Cardiodoron ${ }^{\circledR}$ spürbar verbessert werden. Sowohl bei der Beschwerden-Liste als auch beim PSQI zeigte sich erneut die Tendenz, dass Patienten mit außergewöhnlichen Arbeitszeiten stärker von der Cardiodoron ${ }^{\circledR}$-Einnahme zu profitieren scheinen. Allerdings war der Gruppenunterschied bei diesen beiden Parametern aufgrund der geringen $\mathrm{N}-$ Zahlen nicht signifikant.

Insgesamt 92\% aller Patienten sprachen auf die Therapie mit Cardiodoron ${ }^{\circledR}$ an und erklärten, im Mittel nach rund 2 Wochen einen ersten Eintritt der Wirksamkeit festzustellen. Auch Berichte aus der Praxis bestätigen, dass Cardiodoron ${ }^{\circledR}$ meist innerhalb weniger Wochen zu einer Beschwerdebesserung führt [33, 34, 37, 41]. Der behandelnde Arzt sollte daher dem Patienten verständlich machen, dass Cardiodoron ${ }^{\circledR}$ kein klassisches Schlafmittel ist, sondern etwa 2 Wochen lang eingenommen werden muss, bis eine erste Wirkung eintritt.

Die Verbesserung der beschriebenen Parameter spiegelt sich auch in der übergreifenden Beurteilung der Wirksamkeit wider, die zu rund $90 \%$ bei «sehr gut/gut» lag. Eine nahezu identische Beurteilung der Wirksamkeit (89\% «sehr gut/gut») ermittelte bereits vor über 20 Jahren Weckenmann [27] in einer Ärzteumfrage.

Ein weiterer Vorteil von Cardiodoron ${ }^{\circledR}$ ist die «sehr gute/gute» Verträglichkeit (98\%) verglichen mit Nebenwirkungen allopathischer Medikamente zur Behandlung von Schlafstörungen [20, 24, 63]. Weitere Erfahrungsberichte wie auch diverse Studienergeb- nisse bestätigen durchgehend die gute Verträglichkeit von Cardiodoron $^{\circledR}[17,26-39,64,65]$, die sicher auch ein Grund für die Akzeptanz seitens der Patienten ist und zu einer Compliance führt, die zu 91\% mit mindestens gut eingeschätzt wurde.

Um sicherzugehen, dass keine anderen Maßnahmen zu einer Verbesserung des Krankheitsbildes geführt haben, wurden die Begleittherapien näher betrachtet: Für die 220 der 380 Patienten mit Schlafstörungen, bei denen weitere Maßnahmen ergriffen wurden, waren es vor allem Herz-Kreislauf-Mittel und sonstige sehr unterschiedliche therapeutische Maßnahmen. Klassische allopathische Schlafmittel wurden sowohl in der Dokumentation durch den Arzt als auch durch den Patienten mittels PSQI nur maximal 15-mal genannt. Somit ist davon auszugehen, dass der positive Effekt auf das Befinden bzw. die Schlafstörungen der Patienten tatsächlich Cardiodoron ${ }^{\circledR}$ zugeschrieben werden kann, wobei eine abschließende Beurteilung ohne Kontrollgruppe nicht möglich ist. Das Gebiet der schlafmedizinischen Erkrankungen ist zudem umfangreich [20, 61]. Das Patientenkollektiv der hier vorgestellten Studie erfasste nur einen Ausschnitt daraus, und es wäre von Interesse, darüber hinaus weitere Daten zu sammeln.

Dennoch kann aufgrund der homogenen Ergebnisse dieser Studie gesagt werden, dass Cardiodoron ${ }^{\circledR}$ ein Arzneimittel ist, das ohne nennenswerte Nebenwirkungen bei Schlafstörungen allein oder in Kombination mit funktionellen Herz-Kreislauf-Beschwerden mit Erfolg eingesetzt werden kann. Anders als Arzneimittel mit schlaferzwingendem Charakter scheint Cardiodoron ${ }^{\circledR}$ auf funktionaler Ebene heilend zu wirken, indem es die vegetativen Rhythmen in Einklang bringt. Allerdings muss die Bereitschaft vorliegen, einen gewissen Zeitraum bis zum Eintritt einer spürbaren Verbesserung der Beschwerden in Kauf zu nehmen und auf eine sofortige Wirkung, die den allopathischen Schlafmitteln zu eigen ist, zu verzichten.

\section{Disclosure Statement}

Die Autoren erklären hiermit, dass keine Interessenskonflikte in Bezug auf das Manuskript vorliegen. Die Studie wurde durch die Firma Weleda AG, Schwäbisch Gmünd, finanziell unterstützt.

\section{Literatur}

1 Ehrhardt H: Rudolf Steiners Notizbucheintragungen verschiedener HeiImittelrezepturen. Ein pharmazeutischer Verständnisversuch. Der Merkurstab 2013;66: $36-46$.

2 Medizinische Sektion der Freien Hochschule für Geisteswissenschaften am Goetheanum Dornach/Schweiz: Cardiodoron ${ }^{\circledR}$; in Heilmittelangaben Rudolf Steiners 1999/2000.

3 Fachinformation, Cardiodoron ${ }^{\circledR}$ Dilution, Dezember 2009.

4 Gruber J, Breitkreuz T: Druck ohne Ausgleich. Praxisforum 2009;2:20-26.

5 Pfitzer J: Geschenk für das Herz. Praxisforum 2009;3: $18-24$.

6 Zwiauer J: Cardiodoron ${ }^{\circledR}$ aus pharmazeutischer Sicht. Weleda Korrespondenzbl Aerzte 2002;154:14-20.
7 Brettschneider H: Zur Kombinationswirkung der drei Cardiodoron ${ }^{\circledR}$-Pflanzen im Menschen; in Brettschneider H, Göbel T, Schad W, Strüh H-J, Wember V (Hrsg): Tycho De Brahe-Jahrbuch für Goetheanismus. Niefern-Öschelbronn, Selbstverlag Niefern, 1986, pp 121-164, 148-149.

8 Sharifi N, Souri E, Ziai SA, Amin G, Amini M, Amanlou M: Isolation, identification and molecular docking studies of a new isolated compound, from Onopordon acanthium: a novel angiotensin converting enzyme (ACE) inhibitor. J Ethnopharmacol 2013;148:934-939. 9 Blaschek W, Ebel S, Hackenthal E, Holzgrabe U, Keller K, Reichling J, Schulz V: Hagers Handbuch der Drogen und Arzneistoffe - Hyoscyamus/Atropin/Scopolamin. HagerROM 2006 (Monografie auf CD-ROM). Berlin, Springer-Verlag, 2006.
10 Gilani AH, Khan AU, Raoof M, Ghayur MN, Siddiqui BS, Vohra W, Begum S: Gastrointestinal, selective airways and urinary bladder relaxant effects of Hyoscyamus niger are mediated through dual blockade of muscarinic receptors and $\mathrm{Ca}^{2+}$ channels. Fundam Clin Pharmacol 2008;22:87-99.

11 Heidari MR, Heidari M, Eimani G, Sepehri G: Effect of methanolic extract of Hyoscyamus niger L. on the seizure induced by picritoxin in mice. Pak J Pharm Sci 2009;3:308-312.

12 Khan AU, Gilani AH: In vivo studies on the bronchodilatory and analgesic activities of Hyoscyamus niger and Aspalathus linearis. Lat Am J Pharm 2010;29:777-782.

13 Khan AU, Gilani AH: Cardiovascular inhibitory effects of Hyoscyamus niger. Methods Find Exp Clin Pharmacol 2008;30:295-300. 
14 Vesalainen RK, Kaila TJ, Kantola IM, Tahvanainen KU, Juhani Airaksinen KE, Kuusela TA, Eckberg DL: Lowdose transdermal scopolamine decreases blood pressure in mild essential hypertension. J Hypertens 1998; 16:321-329.

15 Vybiral T, Bryg RJ, Maddens ME, Bhasin SS, Cronin S Boden WE, Lehmann MH: Effects of transdermal scopolamine on heart rate variability in normal subjects. Am J Cardiol 1990;65:604-608.

16 Moser M, Frühwirth M, Kenner T: The symphony of life. Importance, interaction, and visualization of biological rhythms. IEEE Eng Med Biol Mag 2008;27:2937.

-17 Cysarz D, Heckmann C, Bettermann H, Kümmell HC: Effects of an anthroposophical remedy on cardiorespiratory regulation. Altern Ther Health Med 2002;8:7883.

18 Rodenbeck A: Biologische Grundlagen des Schlafens und Wachens. Bundesgesundheitsblatt Gesundheitsforschung Gesundheitsschutz 2011;12:1270-1275.

19 Titze O: Erfahrungen und rationale Gesichtspunkte in der Herz- und Kreislauftherapie. Weleda Korrespondenzbl Aerzte 1983;109:20-31.

20 Fintelmann V: Schlaf und Schlafstörungen. Ein Problem unserer Zivilisation. Therapie und Prophylaxe. Stuttgart, Berlin, Johannes M. Mayer \& Co., 1999, pp $17,37-53,71-74$

21 Pillai V, Roth T, Mullins HM, Drake CL: Moderators and mediators of the relationship between stress and insomnia: stressor chronicity, cognitive intrusion, and coping. Sleep 2014;37:1199A-1208A.

22 Titze O: Schlafstörungen. Weleda Korrespondenzbl Aerzte 1985;113:38-41.

23 Saletu B, Löffler H, Gruber D, Mandl M, Gruber G, Klösch G, Brandstätter N, Dantendorfer K, Frey R, Saletu-Zyhlarz G, Metka M, Huber J, Anderer P: Schlaflaboruntersuchungen zur Insomnie bei postmenopausalem Syndrom. J Menopause 1998;5:26-33.

24 Happe S: Schlaf und seine Störungen im Alter. Bundesgesundheitsblatt Gesundheitsforschung Gesundheitsschutz 2011;12:1311-1318

25 Fietze I, Penzel T: Schlafmedizinische Versorgung in Deutschland. Bundesgesundheitsblatt Gesundheitsforschung Gesundheitsschutz 2011;12:1344-1349.

26 Weckenmann M: Die Wirkung von Cardiodoron ${ }^{\circledR}$ B bei Patienten mit orthostatischem Symptomenkomplex. Med Welt 1970;21:515-521

27 Weckenmann M: Regulative Wirkung eines Pflanzenextraktes bei orthostatisch Labilen. Ärztliche Praxis 1973;30:1453-1456.

28 Weckenmann M: Untersuchung über die kurzfristige Wirkung von Cardiodoron ${ }^{\circledR}$ auf rhythmische Parameter im Stehen. Erfahrungsheilkunde 1984;4:230-238.

29 Weckenmann M: Regulative Therapie funktioneller Herz-Kreislauf-Erkrankungen. Therapeutikon 1987;2: 145-151.

30 Frühwirth M, Avian A, Mayrhoffer M, Meisermann T, Schwarz R, Salameh B, Winker R, Petzl D, Grote V, Köhldorfer P, Kelz C, Schwarz K, Schöber M, Moser M: Cardiodoron ${ }^{\circledR}$ reduces complaints in women with a hypotensive symptom complex - results of a randomized double blind placebo-controlled study. Tagungsband 3. Berlin, Wissenschaftskongress Anthroposophische Medizin, 2012.
31 Mayrhoffer M, Frühwirth M, Avian A, Grote V, Köhldorfer P, Kelz C, Moser M, Puswald B: Relief of symptoms in female patients with a hypotensive symptom complex treated with Cardiodoron ${ }^{\circledR}$ - results of a randomized double-blind controlled study. Forsch Komplementarmed Klass Naturheilkd 2007;14(suppl 1):8.

32 Matthiolius H: Cardiodoron ${ }^{\circledR}$. Beiträge zu einer Erweiterung der Heilkunst 1970;3:77-86.

33 Grünewald-Fritsch A: Hypertonie: Weniger Allopathie durch ganzheitliche Therapie. Praxisforum 2011;2:2021.

34 Grünewald-Fritsch A: Hypertoniebehandlung: Naturheilmittel verbessert den Therapieerfolg. Naturamed 2011; Sonderpublikation September 1-3.

35 Weyers G: Unregelmäßigkeiten von Herzrhythmus und Blutdruck. Eine häufige Beratungsursache mit anthroposophischer Therapieoption. Der Allgemeinarzt 2011;13:62.

36 Weyers G: Pulsrasen, Palpitationen, Hypertonie, Kopfschmerzen, Einschlafstörungen - Stressfolgen anthroposophisch beruhigen. Med Trib 2011;51/52:47.

37 Diehl A: Therapie stressbedingter funktioneller HerzKreislauf-Beschwerden. Praxisforum 2011;2:18-19.

38 Sprenger T: Cardiodoron ${ }^{\circledR}$ als antihypertensive Begleitmedikation. Praxisforum 2011;2:22-23.

39 Gründling PW: Komplementärmedizinische Therapie bei Herzinsuffizienz und Hypertonie. Praxisforum 2011;2:24-25.

40 Meyer F: Orthostatische Synkopen im Alter. Ganzheitlich wieder stabil im Alltag. Kassenarzt 2010;7/8:24-25.

41 Großklaus ES: Chronische Schlafstörungen nachhaltig behandeln. Praxisforum 2013;1:12-13.

42 von Zerssen D, Koeller DM: Die Beschwerden-Liste, Manual. Weinheim, Beltz, 1976.

43 Buysse DJ, Reynolds III CF, Monk TH, Berman SR, Kupfer DJ: The Pittsburgh Sleep Quality Index: a new instrument for psychiatric practice and research. Psychiatr Res 1989;28:193-213.

44 Riemann D, Backhaus J: Behandlung von Schlafstörungen. Weinheim, Beltz, 1996

45 Rother C: Anwendung von Cardiodoron ${ }^{\circledR}$ bei Patien ten mit funktionellen Herz-Kreislauf-Beschwerden und/oder Schlafstörungen - Ergebnisse einer prospektiven, nichtinterventionellen Beobachtungsstudie. Forsch Komplementmed 2013;20:334-344.

46 Gasperi S: Das Geheimnis der Quelle von Levico-Vetriolo. Der Merkurstab 2008;61:228-233.

47 Schramm H: Heilmittel der anthroposophischen Medizin. Grundlagen, Arzneimittelportraits, Anwendungen. München, Elsevier, 2009, pp 1-68.

48 Weckenmann M: Cardiodoron ${ }^{\circledR}$ eine kasuistische Betrachtung. Beiträge zu einer Erweiterung der Heilkunst 1981;34:26-38.

49 Gesellschaft Anthroposophischer Ärzte in Deutschland e.V. und der Medizinischen Sektion der Freien Hochschule für Geisteswissenschaft, Dornach, Schweiz: Vademecum Anthroposophische Arzneimittel, 3. Auflage. Der Merkurstab 2013;66(suppl):204-208.

50 Betz M, Cassel W, Köhler U: Schlafgewohnheiten und Gesundheit bei Jugendlichen und jungen Erwachsenen - Auswirkungen von Schlafdefizit auf Leistungsfähigkeit und Wohlbefinden. Dtsch Med Wochenschr 2012;137:A28.
51 Skowasch D, Nickenig G: Funktionelle Herzbeschwer den. DoctorConsult - The Journal. Wissen für Klinik und Praxis 2010;1:e57-e60.

52 S3-Leitlinie «Umgang mit Patienten mit nicht-spezifischen, funktionellen und somatoformen Körperbeschwerden», AWMF-Reg.-Nr. 051-001, Langfassung, pp 9, 21, 34.

53 Margraf J, DeVries-Wehrhahn E, Sonnentag S: Myokardinfarkt, funktionelle Herzbeschwerden und Paniksyndrom. Psychother Psychosom Med Psychol 1991; 41:31-34.

54 Gottschalk C, Scheuermann P, Wiater A: Schlafstörungen im Kindesalter. Bundesgesundheitsblatt Gesundheitsforschung Gesundheitsschutz 2011;12:1303-1310.

55 Egg M, Paulitsch M, Ennemoser Y, Wüstenhagen A, Schwerte T, Sandbichler AM, Fiechtner B, Köblitz L, Prem C, Pelster B: Chronodisruption increases cardiovascular risk in zebrafish via reduced clearance of senescent erythrocytes. Chronobiol Int 2014;31:680-689.

56 Gu F, Han J, Laden F, Pan A, Caporaso NE, Stampfer MJ, Kawachi I, Rexrode KM, Willett WC, Hankinson SE, Speizer FE, Schernhammer ES: Total and causespecific mortality of U.S. nurses working rotating night shifts. Am J Prev Med 2015;48:241-252.

57 Aho V, Ollila HM, Rantanen V, Kronholm E, Surakka I, van Leeuwen WMA, Lehto M, Matikainen S, Ripatti S, Härmä M, Sallinen M, Salomaa V, Jauhiainen M, Alenius H, Paunio T, Porkka-Heiskanen T: Partial sleep restriction activates immune response-related gene expression pathways: experimental and epidemiological studies in humans. PLoS One 2013;8:e77184.

58 Dusik V, Senthilan PR, Mentzel B, Hartlieb H, Wülbeck C, Yoshii T, Raabe T, Helfrich-Förster C: The MAP kinase p38 is part of Drosophila melanogaster's circadian clock. PLoS One 2014;10:e1004565.

59 Mertler M: Funktionelle Herz-Kreislauf-Erkrankungen. Großer Leidensdruck ohne Organerkrankung. COMED 2011;4:52-54.

60 Heitmann J, Cassel W, Ploch T, Canisius S, Kesper K, Apelt S: Messung von Schlafdauer und Schlafqualität. Bundesgesundheitsblatt Gesundheitsforschung Gesundheitsschutz 2011;12:1277-1283.

61 Mayer G: Häufige schlafmedizinische Erkrankungen. Bundesgesundheitsblatt Gesundheitsforschung Gesundheitsschutz 2011;12:1284-1295.

62 Bott V: Anthroposophische Medizin. Eine Möglichkeit, die Heilkunst zu erweitern. Heidelberg, Karl F. Haug Verlag, 1983, pp 58-59.

63 Riemann D, Nissen C: Substanzinduzierte Schlafstörungen und Schlafmittelmissbrauch. Bundesgesundheitsblatt Gesundheitsforschung Gesundheitsschutz 2011;12:1325-1331.

64 Cysarz D, Heckmann C, Kümmell HC: Wirkung von Cardiodoron ${ }^{\circledR}$ auf die kardiorespiratorische Koordination - ein Literaturüberblick. Forsch Komplementarmed Klass Naturheilkd 2002;9:292-297.

65 Kümmell HC, Bettermann H: Ergebnisse rhythmologi scher Untersuchungen von Cardiodoron ${ }^{\circledR}$ an Gesunden. Der Merkurstab 1996;49:361-371. 